\title{
Arbor
}

\section{Madrid, centro de la red de comunicaciones}

\author{
Antonio Gómez Mendoza
}

Arbor CLXIX, 666 (Junio 2001), 343-358 pp.

Con la excepción de Madrid, todas las capitales de la Unión Europea están emplazadas a escasa distancia del mar o a orillas de un gran río navegable. El aislamiento geográfico de Madrid marcó su evolución histórica y el de su entorno. Como capital del Imperio, su emplazamiento en el corazón de la península ibérica le impidió añadir a su función política y administrativa el barniz de un gran emporio mercantil ${ }^{1}$. Frustrado el intento ilustrado de abrir un canal de navegación a través de la Sierra del Guadarrama, lo que habría puesto en contacto a Madrid con el canal de Castilla y, por ende, con Santander, arrieros, acémilas y carromatos deambularon por los caminos polvorientos en larguísimas recuas, convirtiéndose en estampas habituales del paisaje castellano. La conducción de personas y mercancías por esos medios de sangre sobrecargó los precios finales con el consiguiente efecto negativo sobre la demanda de los madrileños. En un intento por mitigar las consecuencias de esa desventaja locacional, las autoridades recurrieron a un sinfin de artificios administrativos que han sido estudiados magistralmente por David Ringrose ${ }^{2}$. A través de los tiempos, la supervivencia de la Villa pendió del hilo de un complejo sistema de privilegios y subsidios que premiaron a los arrieros y carreteros implicados en el suministro de las subsistencias, materias primas y artículos de consumo.

En el Informe sobre la Ley Agraria, Jovellanos abogó por la unión de los grandes ríos peninsulares mediante una red de canales, caminos y carreteras mejoradas para llenar de «abundancia y prosperidad tantas y tan felices provincias» ${ }^{3}$. Con idéntica contundencia, denunció los acentuados contrastes de los precios de las subsistencias en los mercados 
del interior y del litoral. Sin embargo, los escasos avances en la técnica de transporte no ayudaron a satisfacer las ingentes exigencias de una ciudad de las características de Madrid. Alejada de los centros fabriles que surgieron en la periferia, Madrid nunca proporcionó ese gran mercado capaz de garantizarles su continuidad. Lejos de formar una unidad armónica, el mercado español permaneció fragmentado en una yuxtaposición de células con débiles intercambios entre sí. En el largo plazo, aquella desunión lastró el progreso económico en las regiones del interior peninsular pues carecían de la opción exportadora que la vía marítima brindaba a las áreas del litoral. A partir de la segunda mitad del siglo XIX, su situación comenzó a mejorar con la puesta en servicio de las grandes arterias de ferrocarril. Al nuevo medio de transporte, se añadieron pronto otros sistemas igualmente eficaces para movilizar a bajo coste personas, mercancías e incluso información.

En las páginas que siguen, encontrará el lector un breve comentario sobre las oportunidades de expansión demográfica y económica que brindó a la Villa y Corte el hecho de ser el centro de una red de comunicaciones en su sentido más amplio (carreteras, ferrocarriles, locomoción aérea, telégrafos, teléfonos y electricidad) en el tránsito del siglo XIX al XX. El capítulo se divide en dos partes. En la primera, analizo el abanico de beneficios que extrajo Madrid de su posición en el corazón de la red de caminos de hierro. $\mathrm{El}$ enlace directo por vía férrea con las regiones agrícolas y los núcleos fabriles que le abastecían en alimentos, materias primas y manufacturas, abarató sustancialmente los costes. Es más, los madrileños accedieron al viaje en tren, relegando a un segundo plano a la diligencia. En el siguiente epígrafe, me ocupo de otros cinco adelantos técnicos aplicados a las comunicaciones que, a diferencia del ferrocarril, fueron hijos de la segunda industrialización: motorización, locomoción aérea, telégrafo, teléfono y electricidad. En cada uno de esos casos, la Villa y Corte volvió a ocupar el centro de sus redes. Aquella confluencia de caminos en la capital de España, emulando el conocido dicho sobre Roma, deparó unas formidables perspectivas de crecimiento en el primer tercio del siglo XX. Madrid dejó de ser un poblacho depredador para convertirse en una capital moderna, sede de importantes industrias e instituciones mercantiles. En las conclusiones, argumento que aquellos adelantos técnicos habidos en las postrimerías del siglo XIX borraron los costes que, por espacio de tres siglos, implicó la mal calculada elección de Madrid como capital de España. 


\section{Madrid, epicentro de la red férrea}

Por ser conocidos los benéficos efectos del ferrocarril para el desplazamiento de personas y enseres a largas distancias, no es menester insistir en ellos aquí ${ }^{4}$. Indicaré tan sólo que, junto a la mayor baratura de sus tarifas, el ferrocarril aportó una forma de viajar que, en razón de su regularidad, rapidez, seguridad y comodidad, era inédita para la España de mediados del siglo XIX. Redujo considerablemente la duración de los viajes en comparación con el medio alternativo más rápido. Para evaluar las ventajas de la vía férrea, basta considerar que el tren acortó en $2 / 3$ la duración del viaje a Sevilla por medio de las mensajerías aceleradas ${ }^{5}$.

$\mathrm{Al}$ acortar las distancias, el ferrocarril abrió nuevos mercados lo que permitió una mayor división del trabajo $\mathrm{y}$, por ende, una mayor especialización. Ello redundó en una mayor productividad y, en definitiva, en una mejora del bienestar material de los españoles. Quienes se hallaban más perjudicados por las dificultades orográficas - lo que les sucedía a los habitantes del interior peninsular-, se beneficiaron en mayor grado de aquel cambio. Sin embargo, ninguna ciudad aprovechó mejor que Madrid las ventajas del tren. De ahí que no sea exagerado afirmar que la aplicación del vapor a la locomoción terrestre contribuyó a cambiar su faz, convirtiéndola al fin en una plaza financiera, mercantil e industrial de primera entidad. Los primeros compases de esa metamorfosis tuvieron lugar en el primer tercio del siglo XX. Más allá de las beneficios inmediatos que le deparó el nuevo modo de transporte, Madrid extrajo especiales ventajas de la legislación ferroviaria y, en particular, de la Ley de Bases del Ferrocarril promulgada el 3 de Junio de 1855.

Esta importante disposición legislativa que alumbró el tendido de vías férreas a gran escala en nuestro país, otorgó prioridad a las líneas que, partiendo precisamente de Madrid, terminasen en las costas o fronteras del Reino (Art. $2^{\circ}$ del Capítulo I). Al tratarse de líneas de primer orden y de servicio general, fueron las primeras en ser tendidas por las compañías concesionarias contando para ello con el apoyo del Estado. En sólo diez años, esas líneas formaron una red radial de tipo arborescente con un punto nodal en Madrid. No viene al caso analizar si una red con otra configuración habría satisfecho mejor las necesidades reales de tráfico de la economía española en la segunda mitad del siglo XIX ${ }^{6}$. En esta ocasión, interesa subrayar simplemente que Madrid se convirtió en la ciudad española conectada por vía férrea con el mayor número de entidades de población. En 1900, los madrileños 
podían viajar sin la necesidad de efectuar penosos trasbordos a todas las capitales de provincia, a los principales puertos de mar y a las fronteras y desde allí podían proseguir viaje a Lisboa y París. Un cuarto de siglo bastó para dar cumplimiento a lo aprobado en 1855 por las Cortes. A la posibilidad real de efectuar los desplazamientos por vía férrea, se añadió el acortamiento de las distancias gracias a la importante reducción de los precios del transporte. Por idéntico importe, una tonelada de mercancías podía recorrer una distancia ferroviaria doce veces superior a la efectuada por carretera ${ }^{7}$. Tamaño abaratamiento de los precios abrió inmensas oportunidades de crecimiento para la economía de Madrid.

De las cinco grandes redes de vías férreas que se tendieron en España durante la segunda mitad del siglo XIX, Madrid fue cabecera de tres de ellas: la Compañía de los Caminos de Hierro del Norte de España (NORTE), la Compañía de los Ferrocarriles de Madrid, a Zaragoza y Alicante (MZA) y la Compañía de los Ferrocarriles de Madrid a Cáceres y Portugal (MCP). En conjunto, esas compañías prestaban servicio en unos 7.730 kilómetros de vías férreas, un 70 por ciento de la red española en 1900. Disponían de tres grandes estaciones término en el casco urbano destinadas al servicio de viajeros: Príncipe Pío para NORTE, Atocha para MZA y Delicias para MCP. A partir de los años 1880, varias remodelaciones permitieron su ampliación. Para carbones y materiales de construcción, NORTE habilitó unos embarcaderos especiales en la estación del Paseo Imperial, popularmente conocidos como la estación de Las Pulgas. Las restantes mercancías llegadas a Madrid eran descargadas en una veintena de pequeños apartaderos situados a lo largo de la línea férrea de enlace entre las estaciones de Atocha y Príncipe Pío.

Las estaciones de Madrid ocuparon las primeras posiciones del escalafón ferroviario por la importancia de su tráfico de llegada de viajeros y de mercancías. Varias razones lo explican. Primero, Atocha, Delicias y Príncipe Pío-Pulgas se vieron favorecidas por el hecho ya expuesto de ser Madrid la confluencia de las líneas troncales de la red férrea lo que les puso en conexión directa con los principales centros fabriles, puertos de mar y puntos fronterizos por los que se producían los intercambios con el exterior, tal como se observa en el Mapa 1. Segundo, el ferrocarril desbancó con relativa facilidad al tráfico carreteril en la conducción de las mercancías destinadas al mercado madrileño en razón de su propia naturaleza. Con relación al tonelaje, combustibles, materias primas y alimentos, por este orden, representaban el grueso (91 por ciento en 1901 para el caso de NORTE) de las mercancías 
destinadas a las estaciones madrileñas ${ }^{8}$. Por su bajo valor añadido, esos tres grupos de mercancías resultaban especialmente vulnerables al precio del transporte. Ante una rebaja de éste último, los medios de sangre dejaron paso a la tracción de vapor, siendo más rápida esa sustitución en los recorridos largos que en los cortos, es decir en aquellos que conformaban el grueso del tráfico con destino a Madrid.

\section{Mapa 1}

La red ferroviaria hacia 1900



Fuente: R. Cordero y F. Menéndez, «El sistema ferroviario español...» en Artola (1978), I.

A pesar del sobresalto que causaban los accidentes y a pesar de los frecuentes incumplimientos de los horarios, el ferrocarril popularizó el viaje en la segunda mitad del siglo XIX. En precio, rapidez y comodidad, no tuvo rival. La variedad de servicios ofrecidos por las compañías ferroviarias permitió satisfacer el amplio espectro de la demanda en cada tramo de poder adquisitivo. Por idénticas razones a las apuntadas más arriba, el ferrocarril desbancó al coche de colleras, a la diligencia y a la galera a la hora de movilizar a los viajeros. Al igual que sucedió con las mercaderías, la alta velocidad, que estaba reservada a las personas, sus equipajes y al correo, colocó a las estaciones madrileñas en las primeras posiciones del ranking español por la intensidad de su tráfico. Al confluir sobre Madrid las líneas troncales de la red 
férrea, el número de localidades madrileñas con servicio de tren era comparativamente elevado. Por idéntica razón, el número de localidades de alguna entidad que, sin disponer de estación ferroviaria, se encontraban a corta distancia de un apeadero, era igualmente elevado. No menos de 48 localidades madrileñas se hallaban a menos de diez kilómetros de una estación de ferrocarril; otras 34 distaban una veintena de kilómetros. Por contra, en 22 ocasiones los potenciales viajeros habían de recorrer una distancia superior a 50 kilómetros para abordar un tren ${ }^{9}$. Aunque las estadísticas ferroviarias son poco explícitas, el tráfico de viajeros presentó tres señas características que le diferenciaron de la pequeña velocidad. Primero, hubo equilibrio entre el tráfico de entrada y de salida de viajeros, en comparación con el predominio abrumador de las mercancías llegadas a Madrid sobre las que partían de las estaciones de la Villa. Segundo, el grueso de los viajeros procedían o se dirigían a estaciones relativamente cercanas a Madrid. En 1901, por ejemplo, 4,5 de cada 10 viajeros que se embarcaron en los trenes de la estación de Príncipe Pío, viajaron a las localidades de Pozuelo, Las Rozas, Villalba y San Lorenzo del Escorial. Otros tantos hicieron el viaje en sentido contrario ${ }^{10}$. La mayoría (2/3) pagó billete de $3^{\mathrm{a}}$ clase. Con una mayor presencia de viajeros de $1^{\mathrm{a}}$ y $2^{\mathrm{a}}$ clase, El Escorial, San Sebastián y Santander constituyeron destinos preferidos para los veraneantes madrileños. Y, por último, el movimiento de viajeros presentó rasgos de mayor estacionalidad que la pequeña velocidad. El ferrocarril permitió, en efecto, a los madrileños la asistencia a romerías, corridas de toros y procesiones que se celebraban en poblaciones cercanas a la capital. De no haber sido posible viajar en tren, es seguro que, tan sólo una minoría de los asistentes a esos eventos de carácter extraordinario, habría afrontado los sinsabores de un viaje por carretera.

En suma, el ferrocarril brindó nuevas oportunidades a la economía y sociedad madrileñas al relajar el cuello de botella que impedía su expansión en el medio plazo. No es difícil prever las implicaciones de un escenario hipotético en el que hubiera faltado el servicio ferroviario. De haber sido ese el caso, el volumen de transporte exigido por la alimentación y necesidades generales de los 458 mil nuevos habitantes que engrosaron la población de la Villa y Corte entre 1869 y 1920 con un espectacular aumento anual de un 1,9 por ciento - cuadruplicando la tasa de crecimiento demográfico española entre esos mismos años-, habría obligado a movilizar un formidable ejército de hombres y animales de carga y de tiro. De haber persistido el viejo modelo de transporte basado exclusivamente en el acarreo de sangre que, por 
cierto, era incompatible con la extensión del cultivo que siguió a las sucesivas desamortizaciones de la tierra, el lastre secular impuesto por Madrid al resto de la economía española habría empeorado. Por ello, no es exagerado afirmar que la expansión económica del primer tercio del siglo XX, estudiada por García Delgado en su capítulo, habría estado seriamente amenazada.

\section{Madrid, émulo de Roma}

Si el ferrocarril arrinconó a los medios de transporte tradicionales, no los eliminó por completo ni en Madrid ni, por supuesto, en el resto de la península ${ }^{11}$. Gran número de arrieros con sus acémilas y sus arcaicos vehículos de transporte continuaron cruzando los fielatos madrileños en plena era del ferrocarril. En realidad, ambos sistemas de transporte fueron complementarios pues cada uno atendió las necesidades de una parte concreta de la demanda global. A ello no sólo contribuyeron los vacíos que dejaron las mallas de vía férrea en muchas regiones españolas sino que lo propició, tal como he explicado, la composición del tráfico de mercancías. Mientras que el tren acaparó los flujos de mercancías pesadas y de bajo precio en los largos recorridos, la tracción de sangre siguió acarreando manufacturas y productos poco voluminosos e imperecederos. En las distancias cortas, en cambio, la carretera e incluso las simples veredas no tuvieron rival. Además de ahorrar los gastos de carga y descarga y el desplazamiento hasta la estación de ferrocarril más cercana, muchos labradores dedicaron gustosos su tiempo libre y sus bestias de carga a transportar mercancías en los meses en los que las faenas agrícolas eran menos intensas.

Por consiguiente, los bajos índices de productividad agrícola permitieron sobrevivir al mismo modelo de transporte que había asegurado el abastecimiento de Madrid durante tres siglos. A ello contribuyó igualmente el hecho de que Madrid fuera el epicentro de una red caminera con marcado carácter arborescente. El Decreto de 1761 sentó las bases de un mapa radial con seis ejes principales que se correspondieron con otras tantas carreteras generales. Por medio de una red de esas características, los legisladores borbónicos intentaron satisfacer objetivos administrativos (correo e información), militares y de orden público (abastecimiento de Madrid). Dejaron a un lado cuestiones de índole puramente económica como la baratura y regularidad de los acarreos ${ }^{12}$. Dando la razón a quienes entonces criticaron el plan por ser excesivamente ambicioso, la penuria presupuestaria del Estado prolongó su 
ejecución más allá de lo esperado. Durante la segunda mitad del siglo XIX, las sucesivas leyes Arteta, Moyano y Toreno permitieron completar a trancas y barrancas la red de carreteras, si bien con menor lujo del planeado inicialmente. Mal diseñadas y peor conservadas por la estrechez presupuestaria que afectó a la Dirección General de Obras Públicas, las seis carreteras nacionales que fueron proyectadas en 1761, pusieron a Madrid en comunicación directa con las capitales de provincia, puertos de mar y fronteras ${ }^{13}$. Resulta innecesario reproducir aquí un mapa de carreteras en torno a 1900 pues su configuración era casi mimética del trazado de la red férrea.

En los albores del nuevo siglo, el efecto combinado de la culminación de ese ambicioso proyecto de obras públicas y de la aplicación de un gran avance técnico fruto de la segunda industrialización, brindó a Madrid nuevas oportunidades para mitigar su desventaja locacional. En efecto, quince años antes (1887), dos ingenieros alemanes, Gottlieb Daimler y Karl Benz, demostraron al mundo, a pesar de trabajar por separado, las virtudes del motor de combustión interna o motor de explosión para la tracción mecánica. Su gesta impulsó un desarrollo imparable de la automoción que conoció elevadísimas tasas de crecimiento en los países más industrializados.

El número de matrículas muestra que la motorización progresó en España con cachaza. Al igual que ocurrió en otros muchos países, arraigó en los años de entreguerras. En efecto, la contienda mundial demostró el potencial del vehículo de motor de explosión para el transporte de tropas, pertrechos y material de guerra. Antes de 1914, la demanda de automóviles se limitó a su vertiente para el sport, por lo que estuvo sólo al alcance de personas con un alto nivel de renta. Siendo esa la pauta general de lo acaecido en España, explicar los distintos comportamientos regionales obliga a buscar factores específicos. En lo concerniente a la motorización, Madrid quedó pronto rezagada frente a Barcelona y a otras capitales de provincia. En efecto, la primera matrícula de Madrid correspondió a un automóvil marca Panhard en el verano de 1907. En el bienio anterior, 71 y $192 \mathrm{au}-$ tomóviles fueron matriculados respectivamente en el conjunto del país. Es más, en el preciso año de 1907, el número de vehículos matriculados fue 704. A mayor abundamiento, el primer fabricante español de automóviles, Hispano Suiza, inauguró sus instalaciones en 1904 en la ciudad condal. Otros talleres (Elizalde y Hereter) siguieron su ejemplo. Sin embargo, hubo que esperar a la posguerra civil para ver una fábrica de camiones en las inmediaciones de Madrid. Ese doble retraso madrileño respondió a dos factores. Por un lado, la escasa presencia 
Madrid, centro de la red de comunicaciones

de talleres mecánicos y metalúrgicos en la capital, en contraste con el caso barcelonés más rico en estas manifestaciones por su larga tradición en el sector textil, explicaron, sin duda, la debilidad de los estímulos para crear industrias vinculadas al automóvil en Madrid. Por otro, el sempiterno alejamiento de los puertos de mar dificultó el abastecimiento de las gasolinas de importación sin las cuales no podían funcionar los motores de explosión.

A pesar de esos comienzos tan azarosos, el automóvil abrió unas perspectivas insospechadas para desterrar al pasado los inconvenientes geográficos de Madrid. Para ello, se requerían dos procesos adicionales aún en ciernes a comienzos del nuevo siglo XX. Primero, una comercialización de productos petrolíferos que se tornó en realidad inmediatamente despues de acabada la guerra mundial. Y segundo, una mejora del estado de las carreteras a lo que contribuyó de forma decisiva la creación en 1926 del Circuito Nacional de Firmes Especiales. Madrid figuró entre las ciudades con itinerarios que fueron declarados preferentes ${ }^{14}$.

La aplicación del motor de explosión a la navegación aérea ofreció idénticas perspectivas de un futuro libre de las cortapisas implícitas en el alejamiento madrileño respecto de los pulmones industriales del país y de los lugares de asueto de los vecinos de la capital. El 17 de diciembre de 1903, los hermanos Orville y Wilbur Wright lograron la increible hazaña de elevar del suelo una máquina voladora. Aquel primer vuelo motorizado y controlado de la historia duró una docena de segundos, lo suficiente para que su ingenio volador recorriese cuarenta metros a una escasa altitud.

En los años siguientes, los avances logrados por la aeronáutica se sucedieron a un ritmo trepidante. Buena parte de la explicación responde al hecho de que, en paralelo con lo ocurrido con el automóvil, los militares acertaron a comprender las posibilidades del avión para la guerra moderna. Si bien la participación del avión fue minoritaria durante la conflagración mundial, los duelos aéreos conmocionaron a la opinión pública de los contendientes. Los militares españoles no quedaron a la zaga. La primera promoción de pilotos de guerra data de 1911. Cuando, dos años más tarde, se constituyó el Servicio de Aeronáutica Militar, España disponía ya de 37 aparatos en estado de vuelo. Para su potencial económico, esa flota aérea no desmerecía de la existente en otros países. En los círculos castrenses, existía el convencimiento de que el «aeroplano [sería] el crucero de los países pobres» ${ }^{15}$.

En su versión española, el tándem aeronáutica-ejército convirtió a Madrid en un centro aeronaútico de primer orden. Por razones de 
estrategia militar, su emplazamiento en el corazón de la península carecía de los inconvenientes expuestos más arriba, por no estar sometido al temor de un bombardeo. El aeródromo de Cuatro Vientos que había albergado al Servicio de Aerostación desde comienzos de siglo XX, se convirtió en un centro dinamizador de la incipiente industria aeronáutica española. En sus inmediaciones, se ubicaron dos importantes fabricantes - Construcciones Aeronáuticas (CASA) y Lóringen los años 1920.

MAPA 2

Red aérea española (1925)



\begin{tabular}{|c|l|}
\hline \multicolumn{2}{|c|}{ Leyenda } \\
\hline Línea n$^{\mathbf{0}}$ & \multicolumn{1}{|c|}{ Etapas } \\
\hline 1 & ABCDEF \\
\hline 2 & GHIJ \\
\hline 3 & IHKBea \\
\hline 4 & INOPQRSTUV \\
\hline 5 & PCXYZa \\
\hline
\end{tabular}

\begin{tabular}{|c|l|}
\hline 6 & abcLK \\
\hline 7 & EfXgE \\
\hline 8 & bdehC \\
\hline 9 & HijC \\
\hline 10 & Elm \\
\hline
\end{tabular}


Los estrechos lazos de la industria aeronáutica con la demanda militar y la preferencia del ejército por Madrid como base de sus operaciones en este terreno explican por qué Madrid se erigió en un importante punto nodal de la red aérea española. En clara simbiosis con los proyectos de red caminera y ferroviaria del siglo XIX, el Directorio Militar clasificó las líneas de comunicación aérea en dos grupos, según fueran de servicio general o particular. Imbuida de la doctrina nacionalista propia del momento, la Comisión Interministerial creada por R.O. de 21 de junio de 1924 defendió que el trazado de las líneas aéreas fuera competencia exclusiva del Estado ${ }^{16}$ A ese respecto, los comisionados establecieron un total de diez líneas aéreas. Según se puede observar en el Mapa 2, cuatro de esas líneas debían cruzar el espacio aéreo de Madrid.

La primera'de esas líneas - ABCDEF - partía de San Sebastián y vía Madrid tenía por destino Sevilla. Pretendieron las autoridades españolas dar a la navegación aérea una proyección internacional al contemplar dos prolongaciones más allá de los límites fronterizos españoles. Hacia el norte, la línea permitiría conectar Madrid con París y Londres; hacia el sur, cruzando el Estrecho de Gibraltar, debía poner en comunicación a Madrid con las localidades marroquíes de Larache, Mogador y Cabo Juby y, desde allí, con las Islas Canarias y Fernando Poó. La segunda línea - GCHIJ_compartía la proyección internacional. Desde sus dos puntos extremos, Cáceres y Barcelona, Madrid se conectaría con Lisboa, Génova y París. A diferencia de esas dos líneas, las dos restantes con escala en Madrid estarían dedicadas exclusivamente al tráfico aéreo doméstico. El tercer eje -PCXYZ- debía enlazar Valencia con La Coruña vía Cuenca, Salamanca y Vigo. Desde la capital del Turia, se establecería una prolongación a las Islas Baleares. La cuarta y última línea con escala en Madrid - bdehC- debía cubrir el servicio aéreo en la submeseta norte enlazando entre sí Valladolid, León, Oviedo y Gijón.

De la comparación de los mapas 1 y 2, se desprende que la red de comunicaciones aéreas debía combinar cuatro líneas radiales centradas en Madrid con seis líneas transversales. Por consiguiente, la navegación aérea consiguió un equilibrio territorial del que carecieron la red viaria y la red de caminos de hierro. A pesar de que la Ley General de Ferrocarriles de 1877 promovió el tendido de líneas transversales, las dificultades económicas que conocieron las compañías concesionarias, situación a la que se añadió la falta de recursos del erario público, impidieron su construcción. Por esa razón, los usuarios del avión pudieron volar de Barcelona a Sevilla o a La Coruña sin la 
necesidad de hacer escala en Madrid. Peor suerte les cupo a los usuarios del tren obligados a efectuar trasbordos en las estaciones de Madrid por estar servidos esos destinos por compañías distintas.

Aunque los contemporáneos auguraron a la locomoción aérea un «porvenir vastísimo e insospechado hasta por los mismos genios a quienes se debía la conquista del aire», lo cierto es, no obstante, que la aviación tardó en arraigar en nuestro país ${ }^{17}$. El atraso general de la economía española impidió un rápido despegue del nuevo medio de comunicación. Amén de su aplicación militar ya comentada, la aviación civil quedó restringida al transporte de correo y, en menor medida, a manifestaciones deportivas. Fue decisiva, a ese respecto, la acción pionera del Estado al establecer el Servicio Postal Aéreo por Real Decreto de 17 de Octubre de 1919.

Con relación a la transmisión de correspondencia y, en sentido más amplio, a la transmisión de la palabra y de la información, Madrid sacó provecho a su situación central en las redes telegráfica y telefónica españolas, tal como lo afirma Angel Bahamonde ${ }^{18}$. Al igual que en los casos expuestos anteriormente, el gobierno progresista dotó de una estructura radial a la red telegráfica. Tras el éxito obtenido por la entrada en servicio de la línea Madrid-Irún inaugurada en 1855, las Cortes promulgaron una ley general con el propósito de construir un sistema de líneas radiotelegráficas capaz de poner en comunicación a la Corte con todas las capitales de provincia y departamentos marítimos así como con las fronteras de Francia y Portugal. En tan sólo ocho años, se tendió la estructura básica de esa red que formaron diez mil kilómetros de hilos de cobre y 194 estaciones. A la altura de 1900, esas cifras habían aumentado a 32.500 kilómetros y 1.491 oficinas de telégrafos ${ }^{19}$. En paralelo con lo sucedido en el ferrocarril, Madrid volvió a suministrar el grueso del tráfico con cerca de un tercio de los telegramas emitidos y recibidos. Lo que significó una ventaja indudable para los madrileños por el acortamiento de las distancias telegráficas, se convirtió en un serio inconveniente para las restantes poblaciones. En efecto, la falta de un enlace directo obligó a los telegramas expedidos desde ciudades como Barcelona, La Coruña o Sevilla a transitar por Madrid. A comienzos del siglo XX, la red sufrió las consecuencias derivadas de una fuerte intensificación del flujo telegráfico. El aporte de nuevos recursos públicos evitó caer en un cuello de botella pues se tendió una red de malla para complementar la red radial que había sido construida en la segunda mitad del siglo XIX.

A diferencia del notable éxito que alcanzó el telégrafo gracias a la prontitud con la que se abrió su red, el teléfono se vió prisionero 
de una turbia batalla política entre liberales y conservadores. Mientras los primeros se declararon firmes defensores de la naturaleza privada de las concesiones telefónicas, los segundos, en cambio, se mostraron proclives a reservar la explotación del servicio al Cuerpo de Telégrafos. En 1891, Silvela zanjó el conflicto mediante la implantación de un sistema mixto según el cual el Estado se comprometía a construir las líneas que serían concedidas a particulares. Para ello, dividió el territorio en cuatro zonas delimitadas por otros tantos haces que irradiaban desde Madrid en dirección a los cuatro puntos cardinales. Tan salomónica solución no dejó completamente expedito el camino y el proyecto no cuajó ${ }^{20}$. Sin embargo, aquel fracaso no impidió la creación de algunas compañías de renombre. En 1894, se constituyó la Compañía Peninsular de Teléfonos. Tras la adquisición de varias redes urbanas, entre ellas la madrileña controlada hasta entonces por la Sociedad de Teléfonos de Madrid, la Peninsular formó una nueva empresa - Compañía Madrileña de Teléfonos - para su explotación en 1895. Con esa operación, la Peninsular inició un ambicioso programa de interconexión de redes urbanas que le permitió en 1907 enlazar Madrid con la zona norte y levantina ${ }^{21}$.

La difusión del telégrafo y, en mayor medida, del teléfono exigió una adecuada alimentación eléctrica. A falta de una batería central, cada aparato telefónico debía disponer de pila y magneto, lo que encarecía la instalación y dificultaba su empleo ${ }^{22}$. De ahí la importancia que entrañó para la economía y sociedad madrileñas del cambio de siglo la llegada a su mercado de grandes masas de fluido eléctrico en condiciones atractivas de precio. Por supuesto, la trascendencia de la electricidad sobrepasó ampliamente los estrechos márgenes del transporte de palabras por hilos telefónicos y telegráficos pues significó ni más ni menos una liberación respecto a una dependencia secular de los combustibles fósiles y vegetales. Recuérdese que carbones minerales y leñas constituyeron una de las principales partidas del tráfico de entrada en la Villa y Corte por vía férrea. Al tratarse de productos baratos, su precio final en el mercado resultaba excesivo por los costes del transporte, razón por la cual su demanda progresó a ritmo relativamente bajo.

A principios de los años 1890, vieron la luz las primeras empresas eléctricas en Madrid - La Compañía General Madrileña de Electricidad y The Electricity Supply Company for Spain Ltd-, ambas con capital extranjero. Se les unieron poco después otras pequeñas generadoras. A caballo del nuevo siglo, se desató una fuerte competencia entre esas empresas lo que redundó en provecho de los consumidores. Por ser 
todas esas empresas termoeléctricas, sus exigencias de combustible fósil no aliviaron en absoluto los problemas del transporte ferroviario hacia la capital. La situación no comenzó a cambiar hasta 1907 con la creación de dos empresas hidroeléctricas —Sociedad Hidroeléctrica Española y Salto de Bolarque - para aprovechar la energía hidráulica de varios saltos en los ríos Jucar y Tajo en su confluencia con el Guadiela ${ }^{23}$. La llegada de la hidroelectricidad provocó convulsiones de hondo calado en la economía madrileña. Como es natural, el traslado de la electricidad implicó el tendido de las primeras líneas de transporte a larga distancia. Aunque hubieron de transcurrir muchos años antes de ver completada una red eléctrica a escala nacional, lo cierto es que la red de Madrid con sus ramificaciones hacia el sureste fue, en opinión acertada de Albert Carreras, el «fragmento que más se pareció a un atisbo de red nacional» ${ }^{24}$.

\section{Felipe II y la segunda industrialización}

En su famoso Hand-book for Spain, Richard Ford achacó a la elección de Madrid como capital gran parte de la responsabilidad por el declive económico de España. Deploró que las sucesivas iniciativas reales para trasladar la capitalidad a Valladolid, Lisboa o Sevilla no hubieran dado mejor fruto. En su opinión, la situación era desoladora a mediados de siglo XIX pues no cabía solución alguna al ser «la enfermedad crónica e incurable» ${ }^{25}$. Con alarde de pruebas cuantitativas, estudios recientes han confirmado los temores del famoso viajero británico de tres formas sucesivas. Primero, ha quedado patente que Madrid desangró a su hinterland, al imponerle durante tres siglos una gravosa servidumbre. Segundo, el abastecimiento de la Villa absorbió cuantiosos recursos económicos a causa de la baja productividad de los medios de transporte por vía terrestre en el Antiguo Régimen. Tercero, las claves que explican por qué un sistema tan rudimentario logró sobrevivir a través del tiempo se hallan en la combinación de dos factores. Por un lado, un complejo entramado de subvenciones abarató la factura del transporte fijando precios artificiales para los acarreos. Y, por otro, la propia agricultura de subsistencia, responsable de los modestos índices de productividad, convirtió a los labradores en paro estacional en transportistas eventuales.

En el largo plazo, sin embargo, un modelo de transporte basado en unas premisas tan precarias imponía un techo a la economía de Madrid que no podía ser fácilmente franqueado. En esas condiciones, 
perpetuó su doble función como centro administrativo y político. Por la peculiaridad de su relieve y de su clima, España quedó al margen de la primera fase de la revolución del transporte. En nuestros vecinos del norte, triunfó la navegación interior gracias a la construcción de una tupida red de canales durante el último tercio del siglo XVIII. La situación fue radicamente distinta a mediados del nuevo siglo. En su segunda fase, esa revolución del transporte consistió en aplicar el vapor a la tracción, un adelanto que resultó compatible con la situación española. En virtud de su capitalidad y de la tradición centralista heredada de los reformadores ilustrados, Madrid sacó doble provecho a la llegada del camino de hierro. A la posibilidad inédita de sustituir recursos orgánicos por combustibles fósiles, añadió la ventaja de ocupar el mismísimo centro de la red férrea. Por esa razón, el ahorro de recursos productivos que facultó el ferrocarril, se acrecentó en el caso de Madrid.

Durante la tercera fase de la revolución del transporte acaecida en las postrimerías del siglo XIX, los nuevos adelantos basados en la electricidad y en el motor de explosión volvieron a ser especialmente complacientes con la Corte. No sólo la palabra viajó a velocidades fantásticas gracias a las redes telegráficas y telefónicas sino que, además, se sentaron las bases para un desarrollo inospechado del transporte por carretera de personas y mercancías. Una vez más, el progreso técnico se alió con la política e hizo que sus efectos beneficiasen a la capital en mayor grado que a otras ciudades españolas. Y es que Madrid volvió a ser el centro de una serie de redes de comunicaciones modernas que expandieron su potencial de crecimiento sin provocar por ello rendimientos decrecientes. El sueño de Felipe II de crear una capital moderna y dinámica comenzó por fin a tomar cuerpo.

\section{Bibliografía citada}

Anes, G. (1995): La Ley Agraria, Madrid.

Artola, M. (ed) (1978): Los ferrocarriles en España, 1844-1943 (Madrid). 2 vols.

Aubanell Jubany, A. M. (1992): "La competencia en la distribución de electricidad en Madrid, 1890-1913" en Revista de Historia Industrial, 2, pp. 143/70.

Bahamonde, A. (1997): "Comunicaciones» en Historia de España de Ramón Menéndez Pidal, Madrid, Vol. XXXIII pp. 519/46.

Calvo Calvo, A. (1998): «El teléfono en España antes de Telefónica (1877-1924)» en Revista de Historia Industrial, 13, pp. 59/80.

CARreras, A. (1988): “España durante la revolución tecnológica» en Ministerio de Industria y Tecnología, España: 200 años de tecnología, Madrid, pp. 101/96 
Comfn, F et al (1998): 150 Años de historia de los ferrocarriles españoles, Madrid.

FoRD, R. (1966): A Hand-book for Travellers in Spain and Readers at Home, London ( $1^{a}$ edición de 1845).

Gómez Mendoza, A. (1982): Ferrocarriles y cambio económico en España, 1855-1913. Un enfoque de nueva historia económica, Madrid.

- (1988): «El viaje en el Madrid de Pérez Galdós», Madrid, pp. 183/202.

- (1989): "Ferrocarril, abastecimientos y mercado nacional: Madrid, 1875-1931" en A. Bahamonde y L.E. Otero (eds), La sociedad madrileña durante la Restauración (1876-1931), Madrid, Vol. I pp. 351/76.

- (1991): “Las obras públicas, 1855-1935» en F. Comín y P. Martín Aceña (eds), Historia de la empresa pública en España, Madrid, pp. 177/204.

- (1997): “Transportes» en Historia de España de Ramón Menéndez Pidal, Madrid, Vol. XXXIII pp. 467/518.

- (1999): "Caballo de sangre, caballo de vapor en el transporte de fines del siglo XIX" en A. Carreras et al (eds), La industrialización y el desarrollo económico de España. Homenaje al Doctor Jordi Nadal, Barcelona, Vol. I, pp. 720/35.

- y LÓPEZ Garcí, S. (1992): «Los comienzos de la industria aeronáutica en España y la Ley de Wolff (1916-1929)» en Revista de Historia Industrial I p. 155/78.

Imedio (1943): Pueblos españoles sin estación férrea, Madrid.

Julí́, S., Ringrose, D.R. y Segura, C. (1995): Madrid. Historia de una capital, Madrid.

Madrazo, S. (1984): El sistema de transportes en España, 1750-1850, Madrid.

RingroSe, D.R. (1970): Transportation and Economic Stagnation in Spain, 1750-1850, Durham.

- (1983): Madrid and the Spanish Economy, 1560-1850, Berkeley. 